\title{
A Perspective on the Host Requirements RFCs
}

Status of This Memo

This RFC is for information only; it does not constitute a standard, draft standard, or proposed standard, and it does not define a protocol. Distribution of this memo is unlimited.

Summary

This RFC contains an informal summary of the discussions and conclusions of the IETF Working Group on Host Requirements while it was preparing the Host Requirements RFCs. This summary has several purposes: (1) to inform the community of host protocol issues that need further work; (2) to preserve some history and context as a starting point for future revision efforts; and (3) to provide some insight into the results of the Host Requirements effort.

\section{INTRODUCTION}

A working group of the Internet Engineering Task Force (IETF) has recently completed and published a monumental standards document on software requirements for Internet hosts [RFC-1122, RFC-1123]. This document has been published as two RFC's: "Requirements for Internet Hosts -- Communication Layers", referred to here as "HR-CL", and "Requirements for Internet Hosts -- Application and Support", referred to here as "HR-AS". Together, we refer to them as the Host Requirements RFCs, or "HR RFCs".

Creation of the Host Requirements document required the dedicated efforts of about 20 Internet experts, with significant contributions from another 20. The Host Requirements working group held 7 formal meetings over the past 20 months, and exchanged about 3 megabytes of electronic mail. The HR RFCs went through approximate 20 distinct drafts.

This group of people struggled with a broad range of issues in host implementations of the Internet protocols, attempting to reconcile theoretical and architectural concerns with the sometimes conflicting imperatives of the real world. The present $\mathrm{RFC}$ recaps the results of this struggle, with the issues that were settled and those that remain for future work. This exegesis has several goals: 
(1) to give the Internet technical community some insight into the results of the host requirements effort;

(2) to inform the community of areas that need further work; and

(3) to preserve some history and context of the effort as a starting point for a future revision.

\subsection{GOALS OF THE HOST REQUIREMENTS RFCS}

The basic purpose of the Host Requirements RFCs is to define the requirements for Internet host software. However, the document goes far beyond a simple prescription of requirements, to include:
(a) a bibliography of the documents essential to an implementor;
(b) corrections and updates to the original standards RFC's;
(c) material to fill gaps in the previous specifications;
(d) limitations on implementation choices, where appropriate;
(e) clarification of important issues and the intent of the protocols; and
(f) documentation of known solutions to recurring problems as well as implementation hints.
Broadly speaking, the Host Requirements working group started from the following goals for Internet host software:
(1) Interoperability
(2) Extensibility
(3) Functionality
(4) Efficiency
(5) Architectural Purity

Of these, interoperability was clearly preeminent, while architectural purity had the lowest priority. It is more difficult to assign relative importance to extensibility, functionality, and efficiency, as it varied from one topic to another.

At a more technical level, the working group pursued a set of general goals that included the following: 
* Discourage hosts from unexpectedly acting as gateways.

* Discourage the use of bad IP addresses.

* Eliminate broadcast storms.

* Discourage gratuitous Address Mask Reply messages.

* Facilitate the use IP Type-of-Service for routing and queueing.

* Encourage implementations of IP multicasting.

* Encourage TCP connection robustness.

* Encourage (mandate!) implementation of known TCP performance enhancements.

* Encourage user interfaces that support the full capabilities of the protocols.

* Encourage more complete implementations of FTP.

* Encourage robust mail delivery

* Discourage the source-routing of mail in the Internet.

* Encourage error logging.

In addition to these general technical goals, the working group decided to discourage the use of certain protocol features: e.g., the IP Stream Id option, ICMP Information Request and Reply messages, the RFC-795 TOS mappings, WKS records in the Domain Name System, and FTP Page structure.

The HR RFC tries to deal only with the software implementation, not with the way in which that software is configured and applied. There are a number of requirements on Internet hosts that were omitted from the HR RFC as administrative or configuration issues.

The HR RFCs contain many, many detailed requirements and clarifications that are straightforward and (almost) noncontroversial.

Indeed, many of these are simply restatements or reinforcement of requirements that are already explicit or implicit in the original standards RFC's. Some more cynical members of the working group refer to these as "Read The Manual" provisions. However, they were included in the HR RFCs because at least one implementation has 
failed to abide by these requirements. In addition, many provisions of the HR RFCs are simply applications of Jon Postel's Robustness Principle [1.2.2 in either RFC].

However, not all issues were so easy; the working group struggled with a number of deep and controversial technical issues. Where the result was a reasonable consensus, then definite, firm recommendations and requirements resulted. We list these settled issues in section 2. Section 2 also lists a number of areas where the HR RFCs fill gaping holes in the current specifications by giving extended discussions of particular issues.

However, in some other cases the working group was unable to reach a crisp decision or even a reasonable consensus; we list these open issues in Section 3. Future discussion is needed to ascertain which of these issues really do have "right answers", and which can reasonably be left as implementation choices. Section 4 contains some other areas that the working group did not tackle but which need further work outside the context of the HR RFCs (although the outcome may be reflected in a future revision). Finally, Appendix I lists specific issues for consideration by a future HR RFC revision effort, while Appendix II lists the issues that are relevant to a revision of the Gateway Requirements RFC.

It should be noted that this categorization of issues is imperfect; a few issues appear (legitimately) in more than one category.

For brevity, we do not attempt to define all the terminology or explain all the concepts mentioned here. For those cases where further clarification is needed, we include (in square brackets) references to the corresponding sections of the HR RFCs.

\section{SETTLED ISSUES}

Here are the areas in which the Host Requirements working group was able to reach a consensus and take a definite stand.

\footnotetext{
- ARP Cache Management [CL 2.3.2.1]

Require a mechanism to flush out-of-date ARP cache entries.

- Queueing packets in ARP [CL 2.3.2.2]

Recommend that ARP queue unresolved packet(s) in the link layer.

- Ethernet/802.3 Interoperability [CL 2.3.3]

Impose interoperability requirements for Ethernet and IEEE 802.3
} 


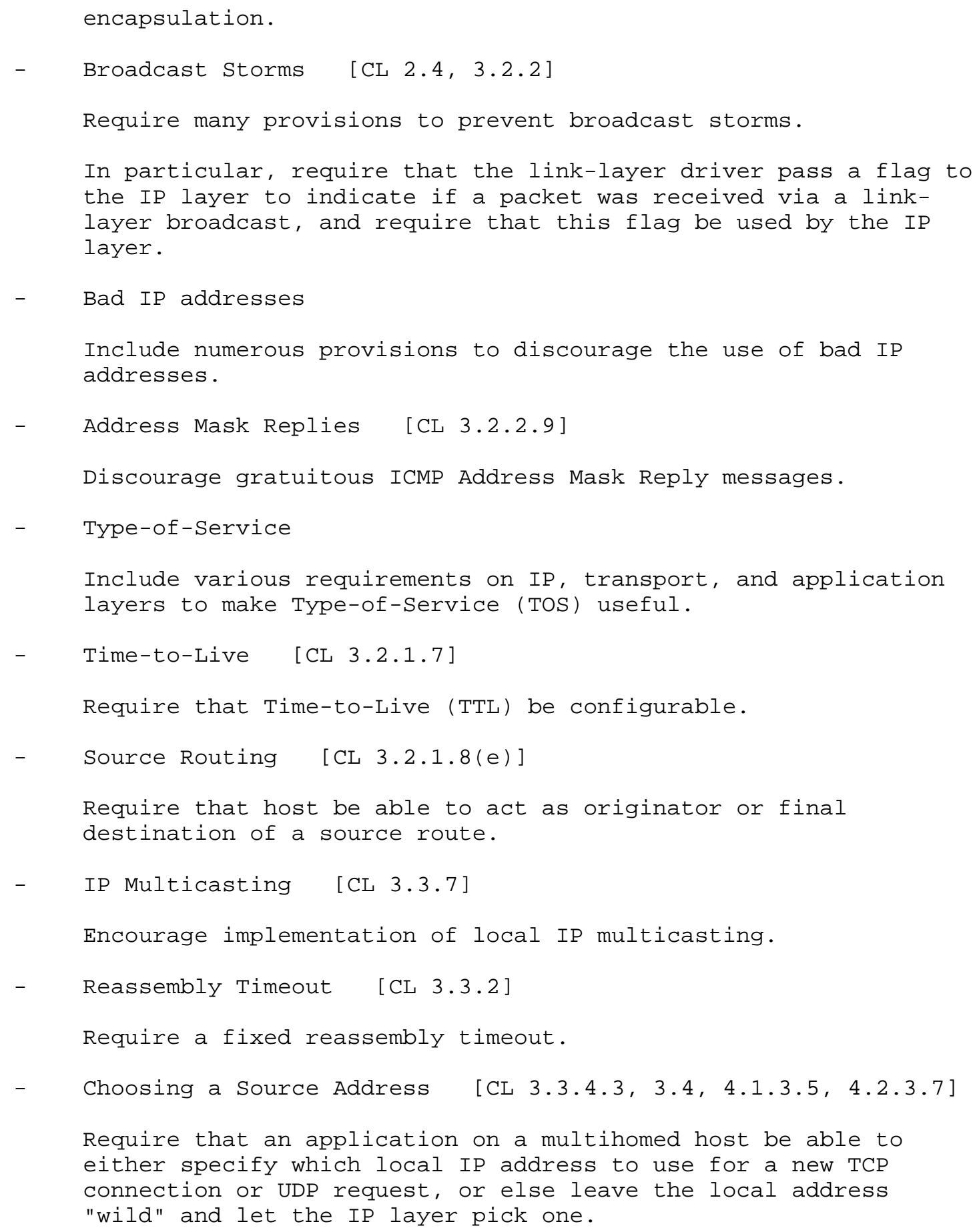




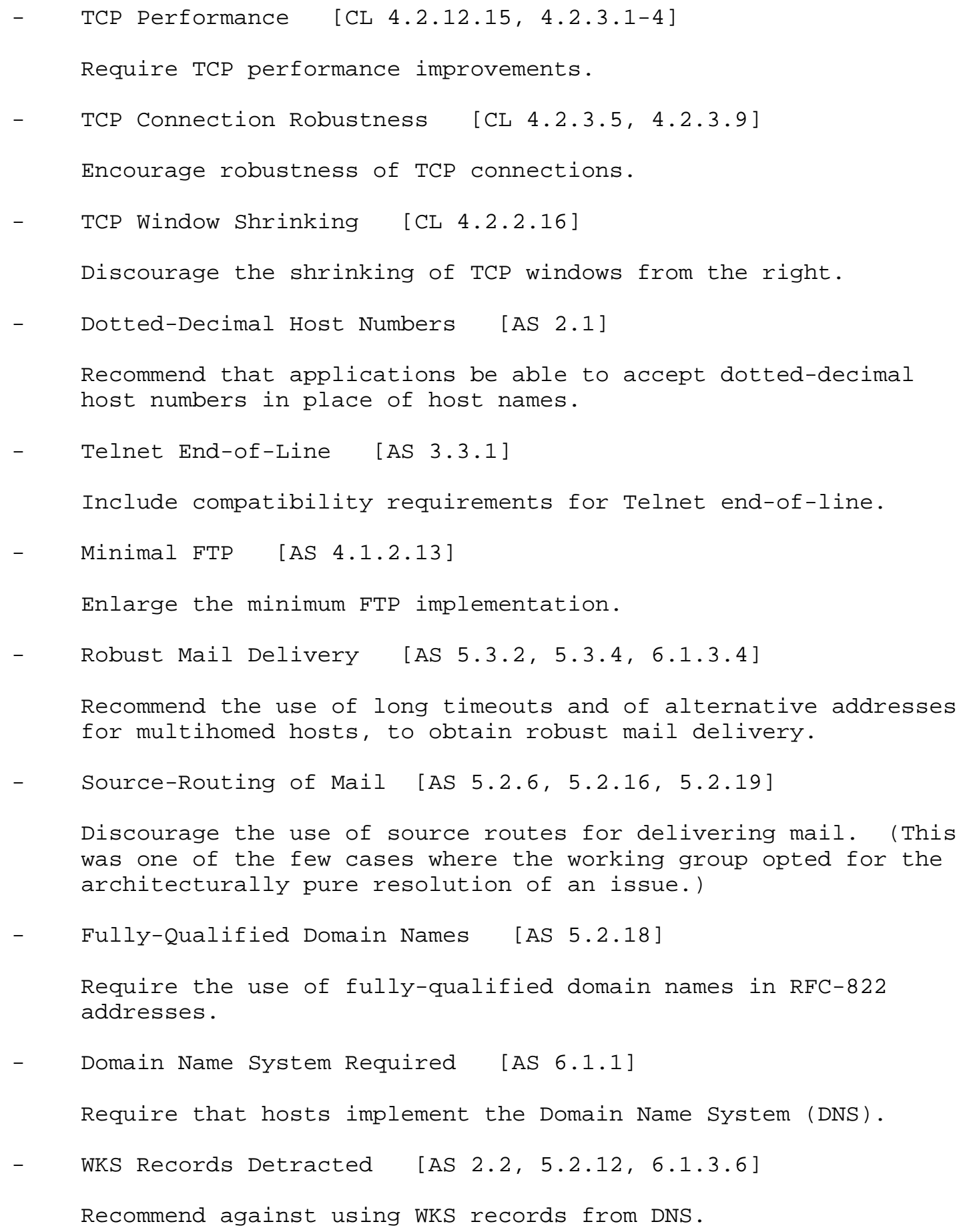




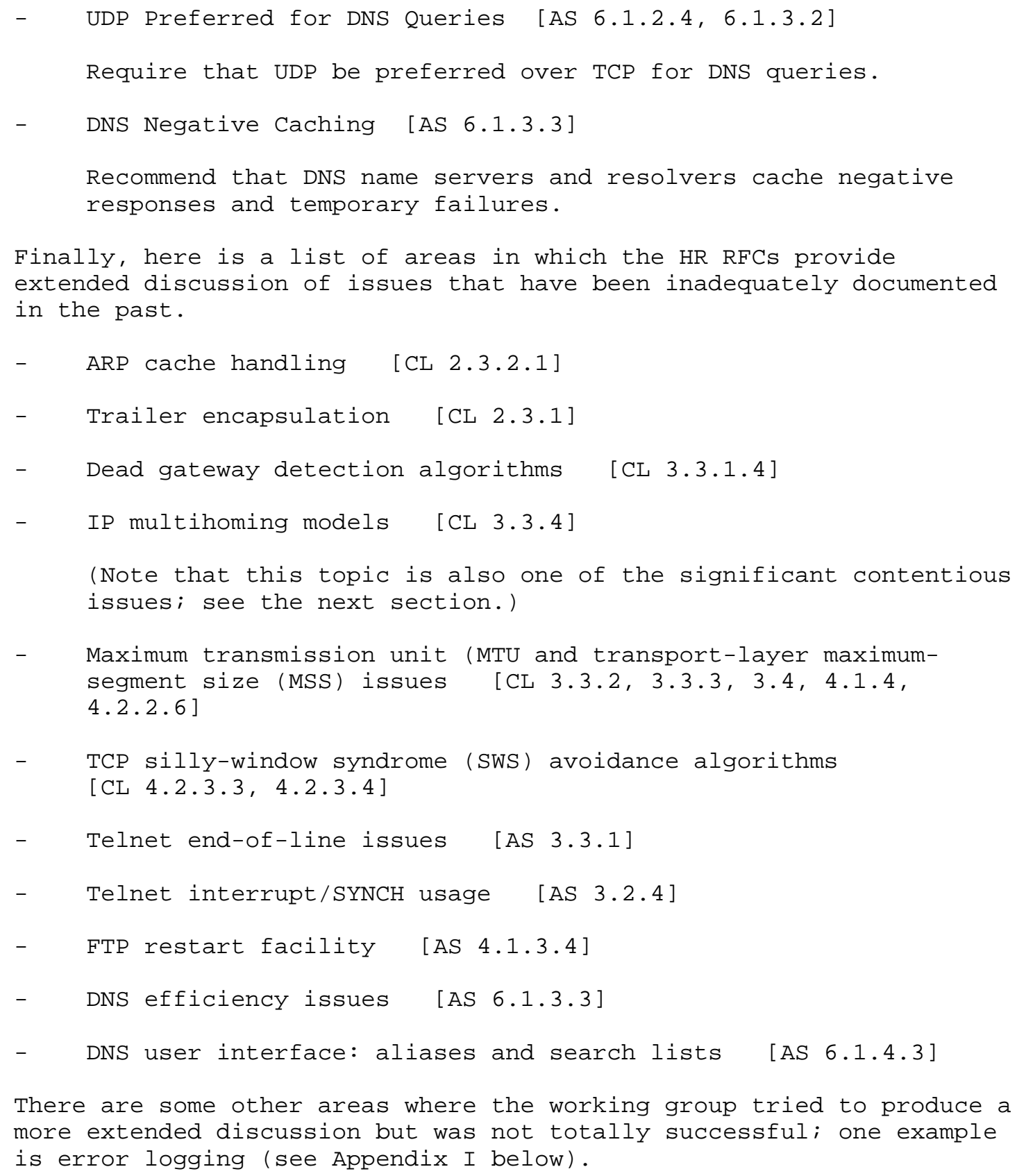


3. OPEN ISSUES

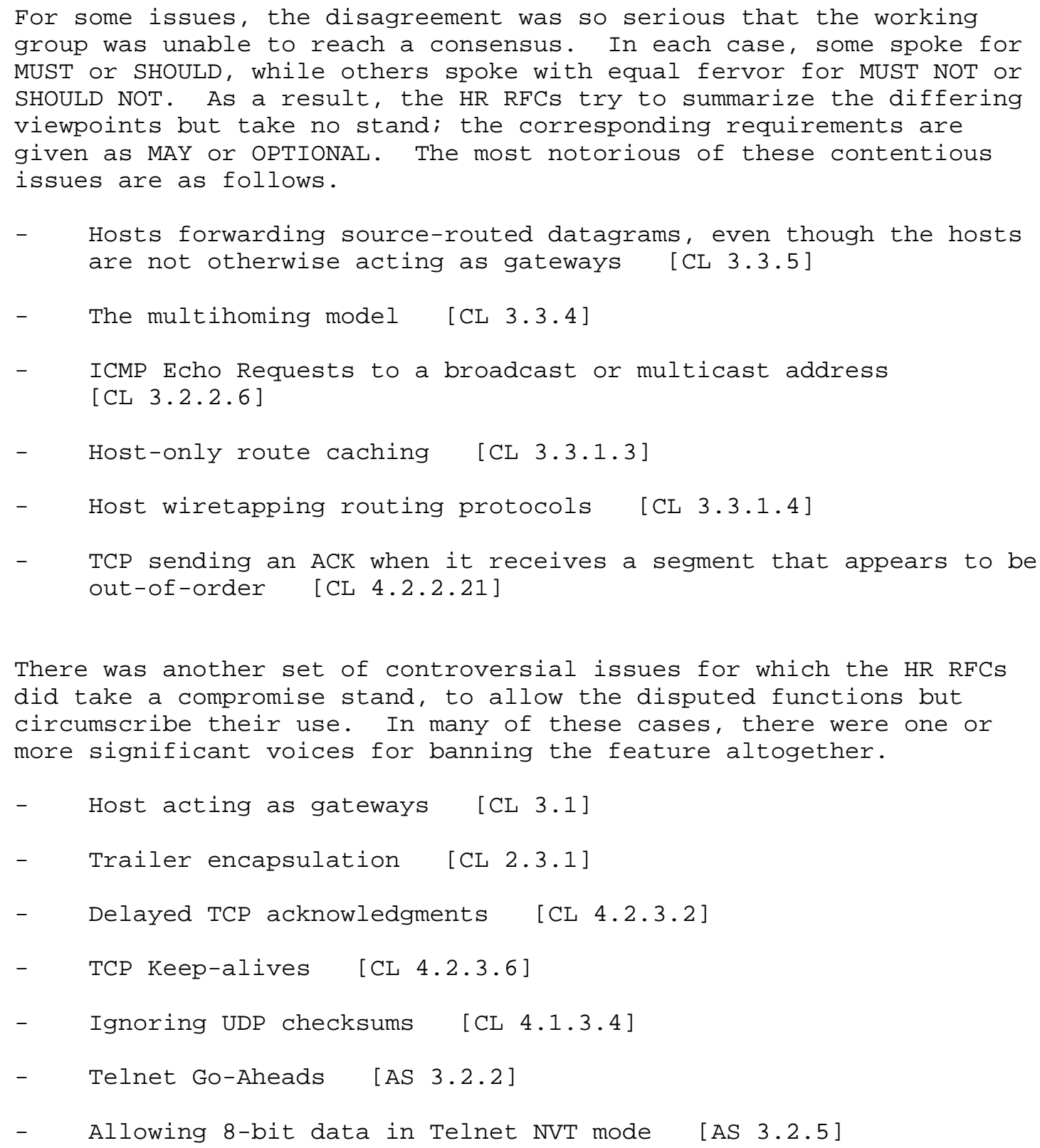


4. OTHER FUTURE WORK

General Issues:

\section{Host Initialization Procedures}

When a host system boots or otherwise initializes, it needs certain network configuration information in order to communicate; e.g., its own IP address(es) and address mask(s). In the case of a diskless workstation, obtaining this information is an essential part of the booting process.

The ICMP Address Mask messages and the RARP (Reverse ARP) protocol each provide individual pieces of configuration information. The working group felt that such piecemeal solutions are a mistake, and that a comprehensive approach to initialization would result in a uniform mechanism to provide all the required configuration information at once. The HR working group recommends that a new working group be established to develop a unified approach to system initialization.

(2)

Configuration Options

Vendors, users, and network administrators all want host software that is "plug-and-play". Unfortunately, the working group was often forced to require additional configuration parameters to satisfy interoperability, functionality, and/or efficiency needs [1.2.4 in either RFC]. The working group was fully aware of the drawbacks of configuration parameters, but based upon extensive experience with existing implementations, it felt that the flexibility was sometimes more important than installation simplicity.

Some of the configuration parameters are forced for interoperability with earlier, incorrect implementations. Very little can be done to ease this problem, although retirement of the offending systems will gradually solve it. However, it would be desirable to re-examine the other required configuration options, in an attempt to develop ways to eliminate some of them.

Link-Layer Issues:

(2) ARP Cache Maintenance

"Proxy ARP" is a link-layer mechanism for IP routing, and its use results in difficult problems in managing the ARP cache.

Even without proxy ARP, the management dynamics of the IP route 
cache interact in subtle ways with transport-layer dynamics; introducing routing via proxy ARP brings a third protocol layer into the problem, complicating the inter-layer dynamics still further.

The algorithms for maintaining the ARP cache need to be studied and experimented with, to create more complete and explicit algorithms and requirements.

FDDI Bit-order in MAC addresses

On IEEE 802.3 or 802.4 LAN, the MAC address in the header uses the same bit-ordering as transmission of the address as data. On 802.5 and FDDI networks, however, the MAC address in the header is in a different bit-ordering from the equivalent 6 bytes sent as data. This will make it hard to do MAC-level bridging between FDDI and 802.3 LAN's, for example, although gateways (IP routers) can still be used.

The working group concluded that this is a serious but subtle problem with no obvious fix, and that resolving it was beyond the scope of the HR working group.

IP-Layer Issues

\section{Dead Gateway Detection}

A fundamental requirement for a host is to be able to detect when the first-hop gateway has failed. The early TCP/IP experimentation was based on the ARPANET, which provided explicit notification of gateway failure; as a result, dead gateway detection algorithms were not much considered at that time. The very general guidelines presented by Dave Clark [RFC-816] are inadequate for implementors. The first attempt at applying these guidelines was the introduction of universal gateway pinging by TOPS-20 systems; this quickly proved to be a major generator of ARPANET traffic, and was squelched. The most widely used implementation of the Internet protocols, 4.2BSD, solved the problem in an extra-architectural manner, by letting the host wiretap the gateway routing protocol (RIP). As a result of this history, the HR working group was faced with an absence of documentated techniques that a host conforming to the Internet architecture could use to detect dead gateways.

After extensive discussion, the working group agreed on the outline of an appropriate algorithm. A detailed algorithm was in fact written down, to validate the discussion in the HR RFCs. This algorithm, or a better one, should be tried experimentally 
and documented in a new RFC.

\section{Gateway Discovery}

A host needs to discover the IP addresses of gateways on its connected networks. One approach, begun but not finished by members of the HR working group, would be to define a new pair of ICMP query messages for gateway discovery. In the future, gateway discovery should be considered as part of the complete host initialization problem.

\section{(6) MTU Discovery}

Members of the HR working group designed IP options that a host could use to discover the minimum MTU of a particular Internet path [RFC-1063]. To be useful, the Probe MTU options would have to be implemented in all gateways, which is an obstacle to its adoption. Code written to use these options has never been tested. This work should be carried forward; an effective MTU choice will become increasingly important for efficient Internet service.

A working group member produced a draft specification for ICMP messages a host could use to ask gateways for routing advice [Lekashman]. While this is not of such pressing importance as the issues listed previously, it deserves further consideration and perhaps experimentation.

Serious connectivity problems have resulted from host software that has too small a TTL value built into the code. HR-CL specifies that TTL values must be configurable, to allow TTL to be increased if required for communication in a future Internet; conformance with this requirement would solve the current problems. However, configurable parameters are an operational headache, so it has been suggested that a host could have an algorithm to determine the TTL ("Internet diameter") dynamically. Several algorithms have been suggested, but considerably more work would be required to validate them. This is a lower-priority problem than issues (4)-(6).

\section{Dynamic Discovery of Reassembly Timeout Time}

The maximum time for retaining a partially-reassembled datagram is another parameter that creates a potential operational headache. 
An appropriate reassembly timeout value must balance available reassembly buffer space against reliable reassembly. The best value thus may depend upon the system and upon subtle delay properties (delay dispersion) of the Internet. Again, dynamic discovery could be desirable.

(10) Type-of-Service Routing in Hosts

As pointed out previously, the HR RFCs contain a number of provisions designed to make Type-of-Service (TOS) useful. This includes the suggestion that the route cache should have a place or specifying the TOS of a particular route. However, host algorithms for using TOS specifications need to be developed and documented.

\section{(11) Using Subnets}

An RFC is needed to provide a thorough explanation of the implications of subnetting for Internet protocols and for network administration.

Transport-Layer Issues:

\section{(12) RST Message}

It has been proposed that TCP RST (Reset) segments can contain text to provide an explicit explanation of the reason for the particular RST. A proposal has been drafted [CLynn].

(13) Performance Algorithms

HR-CL contains a number of requirements on TCP performance algorithms; Van Jacobson's slow start and congestion avoidance, Karn's algorithm, Nagle's algorithm, and SWS prevention at the sender and receiver. Implementors of new TCPs really need more guidance than could possibly be included in the HR RFCs. The working group suggested that an RFC on TCP performance is needed, to describe each of these issues more deeply and especially to explain how they fit together.

Another issue raised by the HR RFCs is the need for validation (or rejection) of Van Jacobson's fast retransmit algorithm.

Application-Layer Issues:

(14) Proposed FTP extensions

A number of minor extensions proposed for FTP should be processed 
and accepted or rejected. We are aware of the following proposals:

(a) Atomic Store Command

The FTP specification leaves undefined the disposition of a partial file created when an FTP session fails during a store operation. It was suggested that this ambiguity could be resolved by defining a new store command, store Atomic (STOA). The receiver would delete the partial file if the transfer failed before the final data-complete reply had been sent. This assumes the use of a transfer mode (e.g., block) in which end-of-file can be distinguished from TCP connection failure, of course.

\section{(b) NDIR Command}

"NDIR would be a directories-only analogue to the NLST command. Upon receiving an NDIR command an FTP server would return a list of the subdirectories to the specified directory or file group; or of the current directory if no argument was sent. ... The existing NLST command allows user FTPs to implement user-interface niceties such as a "multiple get" command. It also allows a selective (as opposed to generative) file-naming user interface: the user can pick the desired file out of a list instead of typing its name." [Matthews]

However, the interface needs to distinguish files from directories. Up to now, such interfaces have relied on a bug in many FTP servers, which have included directory names in the list returned by NLST. As hosts come into conformance with HR-AS, we need an NDIR command to return directory names.

\section{(c) Adaptive Compression}

It has been suggested that a sophisticated adaptive data compression algorithm, like that provided by the Unix "compress" command, should be added as an alternative FTP transfer mode.

\section{(15) SMTP: Global Mail Addressing}

While writing requirements for electronic mail, the working group was urged to set rules for SMTP and RFC-822 that would be universal, applicable not only to the Internet environment but also to the other mail environments that use one or both of these protocols. The working group chose to ignore this siren call, and instead limit the HR RFC to requirements specific to the Internet. 
However, the networking world would certainly benefit from some global agreements on mail routing. Strong passions are lurking here.

DNS: Fully Replacing hosts.txt

As noted in HR-AS [AS 6.1.3.8], the DNS does not yet incorporate all the potentially-useful information included in the DDN NIC's hosts.txt file. The DNS should be expanded to cover the hosts.txt information. RFC-1101 [RFC-1101] is a step in the right direction, but more work is needed.

\section{SUMMARY}

We have summarized the results of the Host Requirements Working Group, and listed a set of issues in Internet host protocols that need future effort.

6. REFERENCES

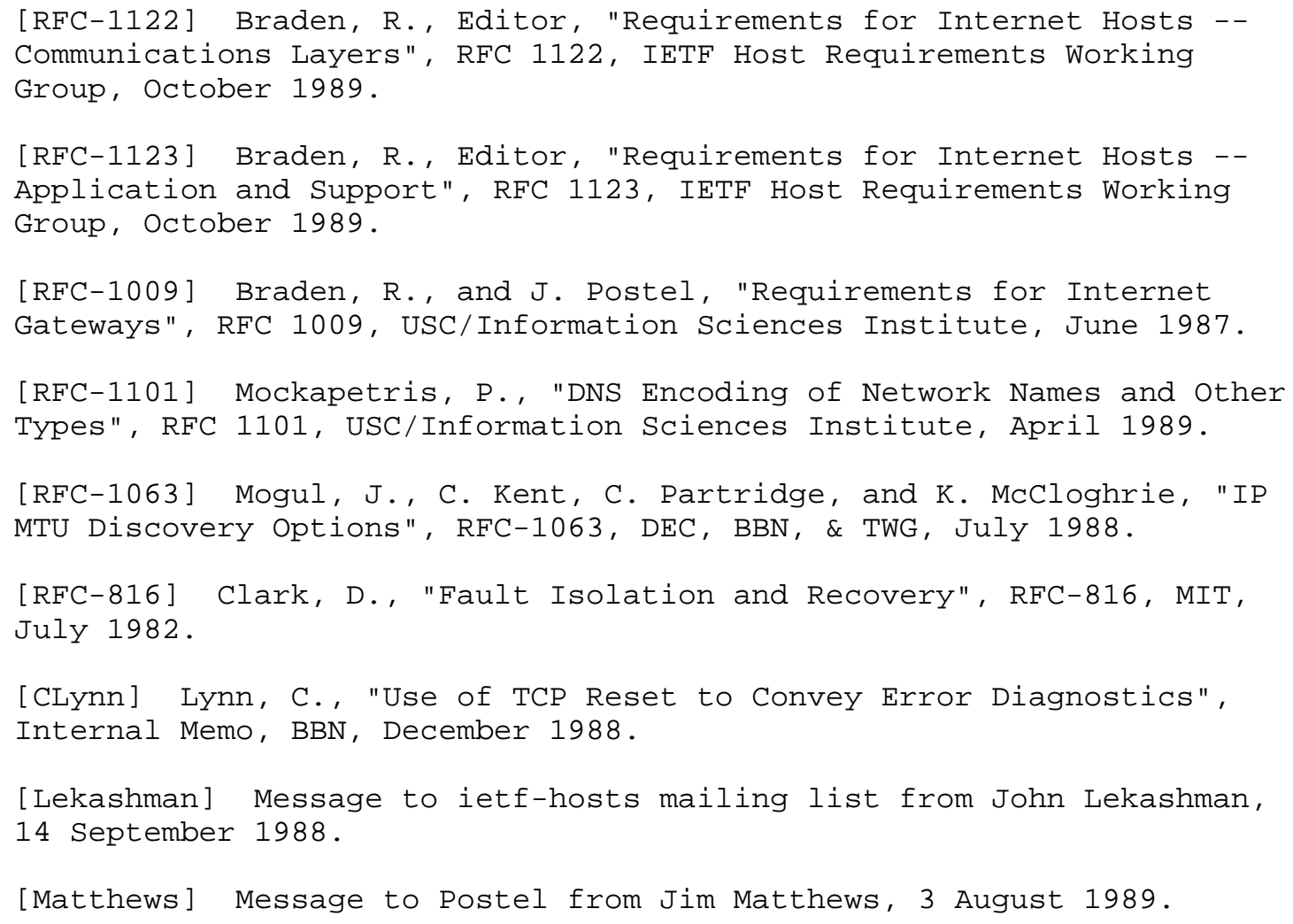


APPENDIX I -- ISSUES FOR FUTURE REVISION

In order to complete the HR RFCs, it was necessary to defer some technical issues. These issues should be considered by the parties responsible for the first update of the HR RFCs.

The issues pending at the time of publication are listed here, in order by protocol layer.

General Issue:

Error Logging

The working group felt that more complete and explicit guidance on error logging procedures is needed than is presently contained in Section 1.2.3 (both HR RFCs).

Link Layer Issues:

- Stolen IP Address

How should a host react when it detects through ARP traffic that some other host has "stolen" its IP address?

IP Layer Issues:

- $\quad$ "Raw Mode" Interface

HR-CL could define an optional "raw mode" interface from the application layer to IP.

- Rational Fragmentation

When a host performs intentional fragmentation, it should make the first fragment as large as possible (this same requirement should be placed on gateways).

- Interaction of Multiple Options

HR-CL does not give specific rules for the interactions of multiple options in the same IP header; this issue was generally deferred to a revision of the Gateway Requirements RFC. However, this issue might be revisited for hosts.

- ICMP Error for Source-Routed Packet

It was suggested that when a source-routed packet arrives with an error, any ICMP error message should be sent with the 
corresponding return route. This assumes that the ICMP error message is more likely to be delivered successfully with the source route than without it.

- $\quad$ "Strong" IP Options and ICMP Types

The HR RFCs takes the general approach that a host should ignore whatever it does not understand, so that possible future extensions -- e.g., new IP options or new ICMP message types -will cause minimum problems for existing hosts. The result of this approach is that when new facilities are used with old hosts, a "black hole" can result. Several people have suggested that this is not always what is wanted; it may sometimes be more useful to obtain an ICMP error message from the old host. To quote Jeremey Siegel:

"The basic premise is that if an option is to have any real meaning at all within an ' [upward] compatible' environment, it must be known whether or not the option actually *carries* its meaning. An absurd analogy might be programming languages: I could make a compiler which simply ignored unknown sorts of statements, thereby allowing for future expansion of the language.

Right now, there are four "classes" of options; only two are defined. Take one of the other classes, and define it such that any options in that class, if unrecognized, cause an ICMP error message. Thus anyone who wants to propose a "strong" option (one which requires full participation by all systems involved to operate correctly) can assign it to that class. Options in the current classes may still be passed through if they are unknown; only "weak" options will be assigned to these classes in the future."

- Network Mask

As explained in HR-CL [CL 3.1.2.3], we believe that a possible future transition for the interpretation of IP addresses may be eased if hosts always treat an IP address as an indivisible 32bit number. However, there are various circumstances where a host has to distinguish its own network number. Charlie Lynn has suggested that indivisibility can be retained if a host is configured with both an address mask (indicating subnetting) and a network mask (with network but not subnet bits).

- WhoAmI Query

The following requirement is needed: for a multihomed host, a 


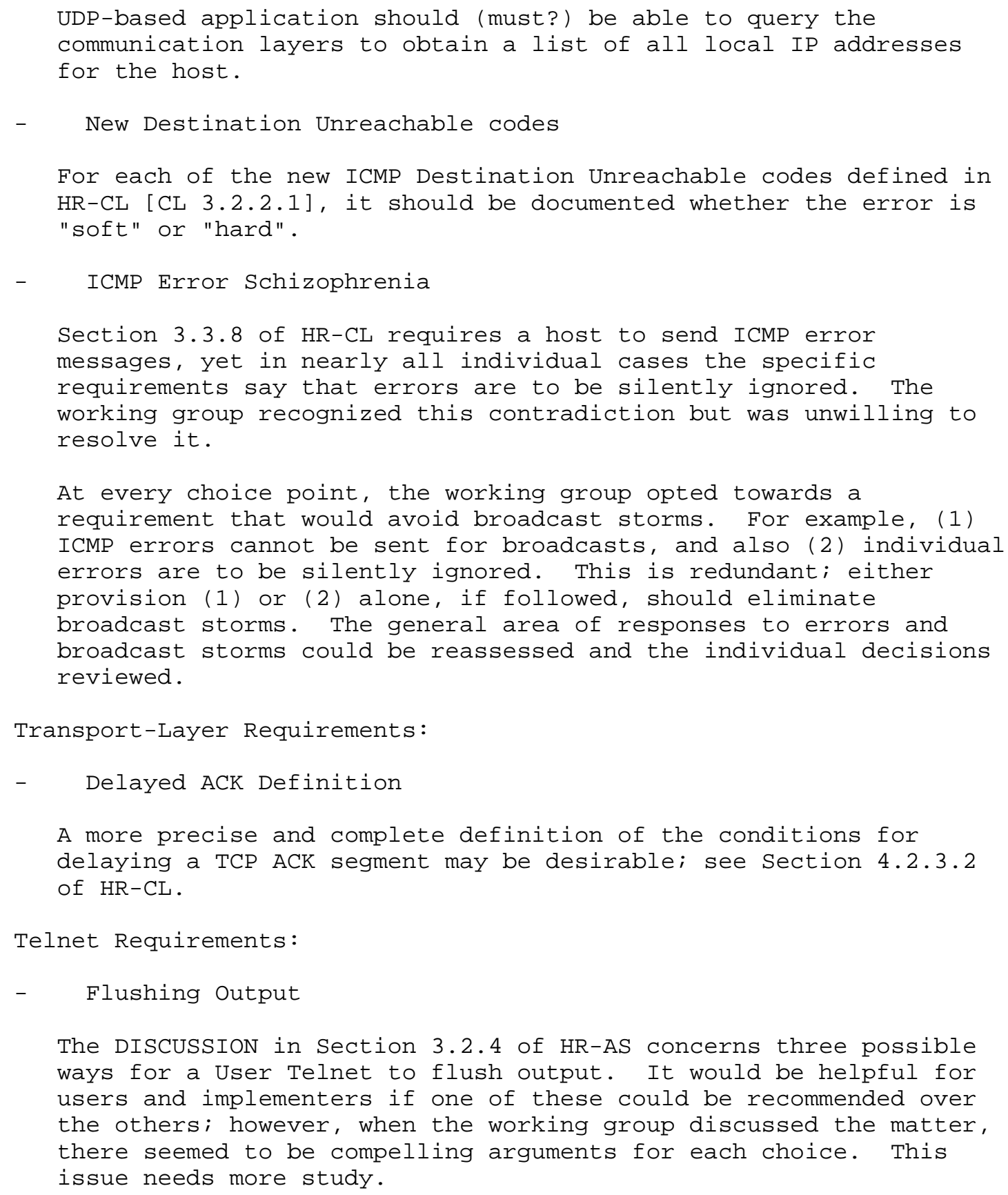


- Telnet LineMode Option

This important new option is still experimental, but when it becomes a standard, implementation should become recommended or required.

FTP Requirements:

- Reply Codes

A number of problems have been raised with FTP reply codes.

(a) Access Control Failures

Note that a 550 message is used to indicate access control problems for a read-type operation (e.g., RETR, RNFR), while a 553 message is used for the same purpose for a write-type operation (e.g., STOR, STOU, RNTO).

LIST, NLST, and STAT may fail with a 550 reply due to an access control violation.

MKD should fail with a 553 reply if a directory already exists with the same name.

(b) Directory Operations (RFC-959 Appendix II)

An RMD may result in a 450 reply if the directory is busy.

Many of the reply codes shown in the text of Appendix II are wrong. A positive completion for CWD should be 250. The 521 code shown for MKD should be 553 (see above), while the 431 shown for CWD should be a 550 .

(c) HELP and SITE Commands

The positive completion reply to a HELP command should be code 214 .

HELP or SITE with an invalid argument should return a 504 reply.

- Bidirectional FTP

The FTP specification allows an implementation in which data transfer takes place in both directions simultaneously, although few if any implementations support this. Perhaps HR-AS should take a stand for or against this. 
SMTP Requirements:

- Offline SEND

Some on the working group felt that the SMTP SEND command, intended to display a message immediately on the recipient's terminal, should produce an error message if delivery must be deferred.

- Header-like Fields

John Klensin proposed:

"Header-like fields whose keywords do not conform to RFC822 are strongly discouraged; gateways SHOULD filter them out or place them into the message body. If, however, they are not removed, Internet hosts not acting as gateways SHOULD NOT utilize or inspect them. Hence address-like subfields of those fields SHOULD NOT be altered by the gateway."

- Syntax of Received: Line

The precise syntax of a revised Received: line (see Section 5.2.8 of HR-AS) could be given. An unresolved question concerned the use of "localhost" rather than a fully-qualified domain name in the FROM field of a Received: line. Finally, new syntax was proposed for the Message Id field.

Appendix II -- Gateway Issues

The working group identified a set of issues that should be considered when the Gateway Requirements RFC [RFC-1009] ("GR RFC") is revised.

- All-Subnets Broadcast

This facility is not currently widely implemented, and HR-CL warns users of this fact. The GR RFC should take a stand on whether or not gateways ought to implement the necessary routing.

- Rational Fragmentation

When a gateway performs intentional fragmentation, it should make the first fragment as large as possible.

- Illegal Source Address

It has been suggested that a gateway should not forward a packet 


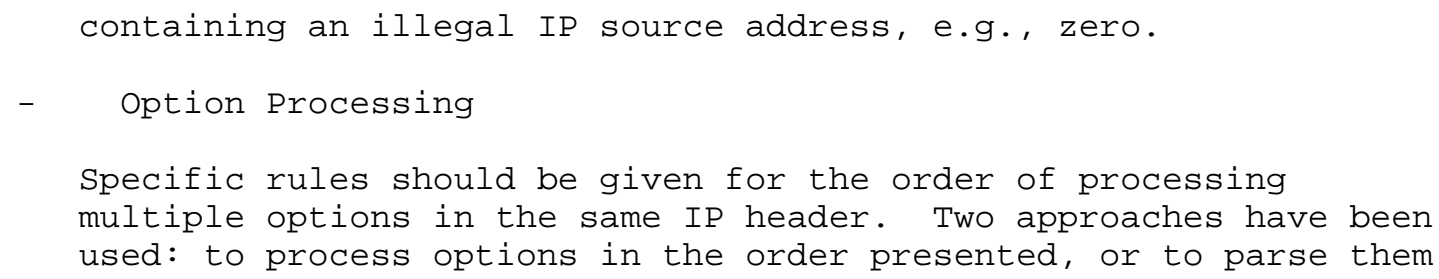

A future revision of the Host Requirements RFCs should incorporate a more complete discussion of security issues at all layers.

Author's Address

Robert Braden USC/Information Sciences Institute

4676 Admiralty Way

Marina del Rey, CA 90292-6695

Phone: (213) 8221511

EMail: Braden@ISI.EDU 